\title{
Amostragem e Abundância de Cigarrinhas e Danos de Dalbulus maidis (DeLong \& Wolcott) (Homoptera: Cicadellidae) em Plântulas de Milho
}

\author{
José M. Waquil ${ }^{1}$ \\ ${ }^{1}$ EMBRAPA/Milho \& Sorgo, Caixa postal 151, 35701-970, Sete Lagoas, MG.
}

An. Soc. Entomol. Brasil 26(1): 27-33 (1997)

Sampling and Abundance of Leafhoppers and Damage by Dalbulus maidis (DeLong \& Wolcott) (Homoptera: Cicadellidae) to Maize Seedling

\begin{abstract}
Sampling leafhoppers on maize (Zea mays), on sorghum (Sorghum bicolor), and on weeds, using a sweep net, indicated that the number of strokes over $10 \mathrm{~m}$ had no effect on the number of insects collected. Dalbulus maidis (DeLong \& Wolcott) was the most common of the leafhoppers collected on maize (93\%), on sorghum (40\%) and on weeds (34\%). The species Peregrinus maidis (Ashmead) was less abundant on all three studied environments. The corn leafhopper $D$. maidis, was more efficiently collected using a plastic bag by covering individual maize plants than using a sweep net. One, five and 10 adults/ plant confined on 10-15 days old plants during a week caused little feeding damage. A quadratic relationship was observed between corn leafhopper infestation density with canopy and root system dry weights of 10-d old plants. The highest level of infestation used (10 adults/plant) reduced $40 \%$ of the canopy and $62 \%$ of the root system dry weight.
\end{abstract}

KEYWORDS: Insecta, vector, corn leafhopper, Peregrinus maidis, Zea mays, Sorghum bicolor.

RESUMO - Em levantamentos de cigarrinhas em milho (Zea mays), sorgo (Sorghum bicolor) e área em pousio, o número de movimentos de rede, entre 5 e 20 vezes por $10 \mathrm{~m}$ de linha, não afetou significativamente o número de insetos coletados. Dalbulus maidis (DeLong \& Wolcott) foi a espécie mais comum entre as cigarrinhas coletadas em plântulas de milho (93\%), sorgo (40\%) e nas áreas em pousio (34\%). A espécie Peregrinus maidis (Ashmead) foi a menos abundante nos três ambientes estudados. A cigarrinha-do-milho, D. maidis, foi coletada mais eficientemente através da amostragem individual de plantas, usando-se o método do saco de plástico, do que com o uso da rede entomológica. Para determinar os danos de D. maidis, um, cinco e 10 adultos/planta foram confinados em plântulas com 10 e 15 dias de idade, durante uma semana. Em plântulas com 15 dias não se verificou efeito significativo. Por outro lado, em plântulas com 10 dias observou-se uma relação quadrática da densidade de infestação com o peso seco da parte aérea e do sistema radicular. No nível máximo de infestação utilizado, 10 adultos/planta, observou-se perdas de $40 \%$ no peso seco da parte aérea e de $62 \%$ no peso seco do sistema radicular.

PALAVRAS-CHAVE: Insecta, vetor, cigarrinha-do-milho, Peregrinus maidis, Zea mays, Sorghum bicolor. 
Levantamentos detectaram mais de 30 espécies de cigarrinhas na cultura do milho, Zea mays, nos Estados Unidos, estando a proporção dessas espécies em função da época e local (Douglas et al. 1966). Dalbuius maidis (DeLong \& Wolcott) foi considerada como uma das principais espécies de cigarrinhas vetoras de patógenos em milho (Nault \& Ammar 1989). A cigamnha D. maidis, além de transmitir os agentes do enfezamento do milho (Kunkel 1946) e viroses, como Rayado-fino (Kitajima 1979), causa dano direto pela sucção de seiva (Bushing \& Burton 1974). No Brasil, Costa et al. (1971) registraram as cigarrinhas D. maidis e Peregrinus maidis (Ashmead) como importantes vetores de patógenos em milho e também a ocorrência da virose Rayadofino em $60 \%$ das plantas, nos plantios tardios no Estado de São Paulo. Perdas de 28,6\% na produção de grãos devido a essa virose foram registradas em Minas Gerais (J. M. Waquil et ai., não publicado). No Vale do São Francisco, A. S. Costa \& E. W. Kitajima (não publicado) relataram a ocorrência de enfezamento em milho e S. Leão Veiga (não publicado), a presença de $P$. maidis, cujos ovos estavam parasitados por Anagrus flaveolus Waterhouse (Hymenoptera: Mymaridae).

Tanto a rede entomólogica como a de varredura, conforme descrita em Gallo et al. \{1978), podem ser usadas para a amostragem de cigarrinhas. Stevens \& Steinhauer (1973) avaliaram a performance e eficiência do D-Vac) como método para amostrar Hypera postica (Gyllenhal) em alfafa, Medicago saliva. Comparando-se a coleta de insetos em forrageiras, através de rede e coleta a vácuo, Callahan $e t$ al. (1966) concluíram que apesar desse método ser mais eficiente, ele gasta o dobro do tempo para amostrar a mesma área. Entretanto, a rede se mostrou ineficiente para a amostragem de ninfas (Simonet et al. 1978). Em alfafa, Cherry et al. (1977) desenvolveram fatores para correção dos dados obtidos através de rede entomólogica, para estimar a densidade da cigarrinha Empoasca fabae (Harris) em função das condições climáticas A rede entomólogica tem sido também utilizada para a amostragem de predadores em algodão, Gossypium hirsutum, (Campos et al. 1986), em tomateiro, Lycopersicum esculentum, (Raga et al. 1990) e a de varredura, para a coleta de insetos em geral no caupi, Vigna unguiculata (Fazolin 1995). Para a amostragem da mosca do sorgo, Contarinia sorghicola (Coquillet), em panícula de sorgo, Sorghum bicolor, Waquil et al. (1986) utilizaram o método do saco de plástico.

Em Sete Lagoas, MG, observou-se uma alta incidência de cigarrinhas em milho semeado tardiamente (fevereiro/março). Este trabalho foi conduzido para estabelecer o melhor método de levantamento da população de cigarrinhas, a predominância de espécies em milho e em sorgo e para estimar a redução do desenvolvimento de plântulas de milho em função da densidade de infestação por $D$. maidis.

\section{Material e Métodos}

Amostragem e Abundância de Cigarrinhas. Num experimento em fatorial, avaliou-se o efeito do número de movimentos de rede (5, 10,15 e 20 por $10 \mathrm{~m}$ ) e tipos de cobertura do solo (milho, sorgo e área em pousio) na incidência de cigarrinhas. Posteriormente, o método da rede entomológica foi comparado com a amostragem individual de plantas com saco de plástico. Este método, adaptado de Waquil et al. (1986), consistiu no ensacamento repentino do cartucho da planta com um saco de plástico e colheita imediata desse cartucho, para posterior contagem dos insetos. Os cartuchos colhidos foram armazenados em freezer a $-15^{\circ} \mathrm{C}$ e, depois de três dias, as amostras foram avaliadas, contando-se o número de insetos/ cartucho. Esses dois métodos de amostragem foram utilizados durante um ano de levantamento, tendo sido analisadas 30 amostras/semana, provenientes de uma área de 300 $\mathrm{m}^{2}$ de milho, semeada mensalmente. Os insetos coletados, depois de separados e quantificados em laboratório, foram enviados a especialistas, para identificação das espécies. 
Danos de D. maidis em Plântulas de Milho. Este ensaio foi conduzido em casa de vegetação, utilizando-se vasos de plásticos com capacidade para $5 \mathrm{~kg}$ de solo, nos quais o milho foi semeado e, posteriormente, desbastado para cinco plântulas/vaso. Num fatorial de $2 \times 4$, estudou-se o efeito de idade das plantas para a infestação (10 e 15 dias) e da densidade de infestação $(0,1,5$ e 10 cigarrinhas/ planta). Foram utilizadas quatro repetições inteiramente ao acaso. Para a infestação, utilizaram-se gaiolas cilíndricas ( $25 \mathrm{de} \longrightarrow$ x $34 \mathrm{~cm}$ de $\mathrm{H}$ ), de armação de arame e cobertura de "voil". Os insetos ficaram confinados sobre plantas durante uma semana, sendo os mortos substituídos diariamente. Quarenta dias após, as plantas foram arrancadas dos vasos, sob jato de água, para evitar a perda de raízes, e obteve-se o peso fresco e seco, separados para as partes aérea e subterrânea da planta. Os resultados foram submetidos a análise de variância e as médias separadas pelo teste de Duncan $(\mathrm{P} \leq 0,05)$.

\section{Resultados e Discussão}

Amostragem e Abundância de Cigarrinhas. Os resultados dos levantamentos, utilizandose diferentes números de movimentos de rede, não mostraram diferenças significativas para as seguintes variáveis: número total de cigarrinhas, número de $D$. maidis e o número de $P$. maidis presentes no milho, no sorgo ou na área em pousio (Tabela 1). Também não foi significativa a interação entre os efeitos dessas coberturas vegetais com o número de movimentos de rede. Entretanto, o tipo de cobertura vegetal afetou significativamente a densidade desses insetos (Tabela 1).

As duas espécies de insetos mais abundantes nos levantamentos foram identificadas como $D$. maidis e $P$ maidis. Nota-se a predominância de cigarrinhas nas áreas com milho e em pousio em relação à área com o sorgo (Tabela 1). D. maidis predominou no milho (93\% dos espécimes), no sorgo (40\%) e na área em pousio (34\%). Entretanto, nesses dois últimos ambientes, a predominância de $D$. maidis sobre $P$. maidis foi menor. A coleta de $D$. maidis no sorgo, não comprova a utilização dessa espécie como hospedeiro. É possível que essa cigarrinha esteja utilizando o sorgo, apenas para alimentação e/ou abrigo, pois são citados como hospedeiros de D. maidis apenas as espécies Z. mays, Tripsacum dactyloides e Euchlaena mexicana (Tsai 1988).

Comparando-se os resultados obtidos com a amostragem individual de plantas e os da rede entomológica, ao longo de um ano, verificou-se que a eficiência da rede em capturar os insetos caiu à medida que a densidade da população, estimada pelo método do saco de plástico, aumentou (Fig. 1). Nos meses de junho a dezembro, a eficiência dos dois métodos foi semelhante, mas nos meses de fevereiro a abril, quando ocorreu o pico populacional de D. maidis, a amostragem individual foi mais eficiente. Esse pico populacional se deve à multiplicação dos insetos nos milhos semeados na primavera/verão. Nas demais épocas, a densidade de $D$. maidis foi baixa, pela falta de hospedeiro e por essa espécie não se desenvolver no inverno. A discrepância entre os dois métodos de amostragem pode ser devido ao fato de que, em áreas com alta densidade de cigarrinha, um maior número de insetos escapem da rede durante os movimentos de coleta. Dessa forma, para se fazer uma análise qualitativa da composição da comunidade dessas cigarrinhas, pode-se utilizar a rede entomológica, mas para se determinar variações quantitativas, deve-se optar pela amostragem individual de plantas.

Danos de $D$. maidis em Plântulas de Milho. As plântulas de milho com 10 e 15 dias de idade respondem diferentemente quando infestadas por D. maidis (Tabela 2). Plântulas com 15 dias, infestadas por diferentes densidades de cigarrinhas, não apresentaram diferença significativa quanto ao acúmulo de matéria seca da parte aérea e da parte subterrânea, mas, nas plântulas com 10 dias, essa diferença foi constatada. Embora não se tenha detec- 
Tabela 1. Média ( \pm EP) do número de cigarrinhas coletadas através de diferentes movimentos de rede entomológica/10 $\mathrm{m}$ de linha e em diferentes coberturas de solo.

\begin{tabular}{|c|c|c|c|}
\hline \multirow[b]{2}{*}{ Tratamentos } & \multicolumn{3}{|c|}{ Número de adultos/10 m } \\
\hline & Cigarrinhas $^{2}$ & D. maidis ${ }^{2}$ & P. maidis $^{2}$ \\
\hline \multicolumn{4}{|l|}{$\mathrm{N}^{\circ}$ mov. Rede/10m } \\
\hline 5 & $47,5( \pm 8,4)$ & $28,7( \pm 10,4)$ & $3,5( \pm 1,3)$ \\
\hline 10 & $42,0( \pm 5,6)$ & $25,3( \pm 8,1)$ & $2,8( \pm 0,8)$ \\
\hline 15 & $45,2( \pm 9,6)$ & $24,8( \pm 7,5)$ & $2,6( \pm 0,7)$ \\
\hline 20 & $49,0( \pm 9,4)$ & $29,8( \pm 7,6)$ & $5,2( \pm 2,2)$ \\
\hline \multicolumn{4}{|l|}{ Cobertura do solo } \\
\hline Milho & $56,3( \pm 2,8)$ a & $52,5( \pm 3,3)$ a & $1,8( \pm 0,4) b$ \\
\hline Sorgo & $23,5( \pm 3,2) b$ & $10,8( \pm 1,3) \mathrm{b}$ & $2,1( \pm 0,6) b$ \\
\hline Ervas Daninhas & $58,0( \pm 4,9)$ a & $19,5( \pm 2,4) b$ & $6,6( \pm 1,4) a$ \\
\hline
\end{tabular}

${ }^{1}$ Não houve diferença significativa no número de insetos coletados para os diferentes números de movimentos de rede.

${ }^{2}$ Médias seguidas pela mesma letra, nas colunas e para o fator cobertura de solo, não diferem entre si pelo teste de Duncan $(\mathrm{P} \leq 0,05)$.

tado diferença significativa entre os níveis de infestação de um e cinco adultos/planta, para linear foi significativa como para o peso fresco algumas das variáveis avaliadas, a regressão de raízes $(\mathrm{r} 2=0,49)$ e o peso fresco da parte aérea (r2-0,32) (Fig. 2). Entretanto, as

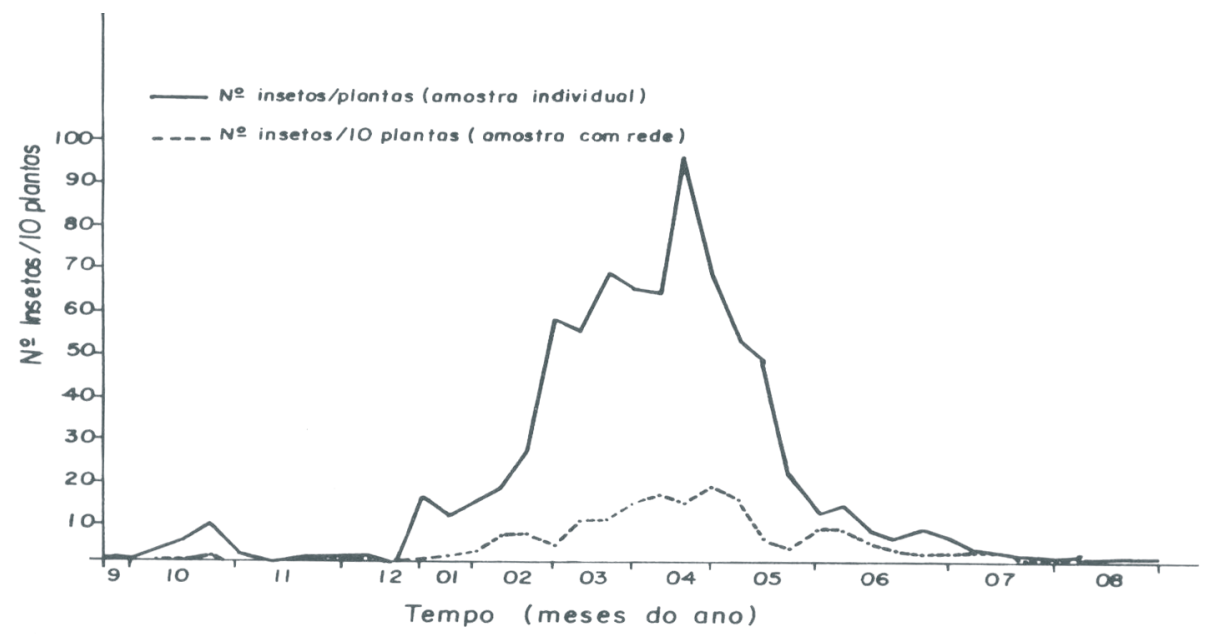

Figura 1. Número médio de cigarrinhas, Dalbulus maidis, obtido em um ano de levantamentos realizados através de rede entomológica e amostragem individual de plantas de milho. 
equações que melhor se ajustaram aos dados foram as quadráticas, que resultaram em maiores coeficientes de regressão, para o peso total das plantas $(\mathrm{r} 2=0,51)$, o peso da parte aérea $(\mathrm{r} 2=0,44)$ e o peso da parte subterrânea $(\mathrm{r} 2=0,55)$. Ao se comparar as variáveis avaliadas nos dois níveis extremos de infestação, a testemunha e a densidade de 10 adultos/plântula (Tabela 2), verificou-se uma redução de $40 \%$ no peso fresco e/ou seco da parte aérea,

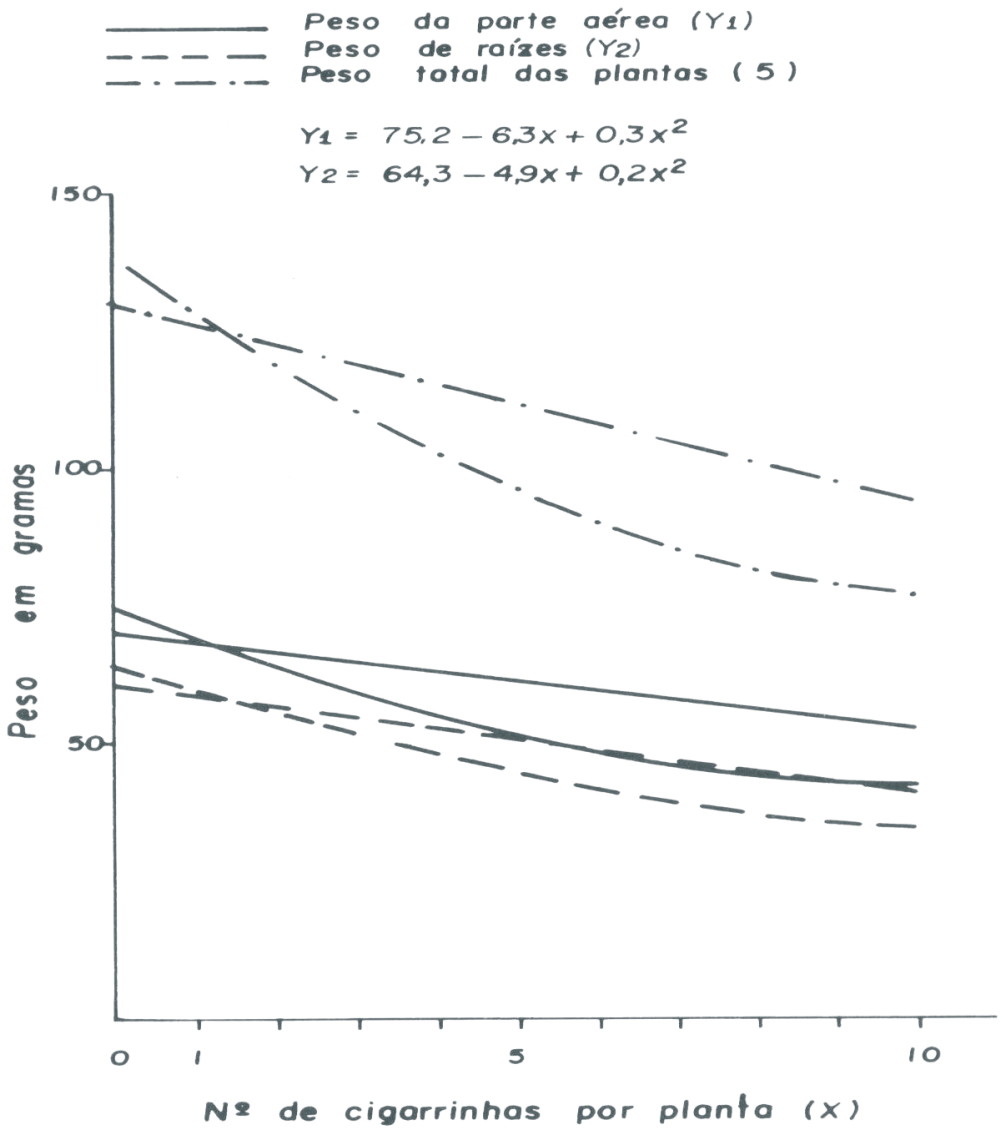

Figura 2. Redução do peso de plântulas de milho devido aos danos da cigarrinha Dalbulus maidis.

$53 \%$ no peso fresco e $62 \%$ no peso seco de raízes. Esses dados estão de acordo com os obtidos por Bushing \& Burton (1974), que encontraram perdas de peso de $34,6 \%$ devido ao ataque de D. maidis, em milho cultivado para silagem, quando compararam o peso seco de parcelas testemunhas com parcelas tratadas com phorate.
Os resultados indicaram que em levantamentos de cigarrinhas, o número de movimentos de rede $5,10,15$ e 20 vezes/ $10 \mathrm{~m}$ de fileira de plantas não afetou o número de insetos coletados e que maior precisão nos levantamentos foi obtida pela amostragem individual de plantas, através do método do saco de plástico. A incidência de cigarrinhas, em geral, foi 
Tabela 2. Médias ( \pm EP) do peso verde e seco, da parte aérea e de raízes, de plântulas de milho infestadas artificialmente pela cigarrinha Dalbulus maidis.

\begin{tabular}{|c|c|c|c|c|c|}
\hline \multirow{2}{*}{$\begin{array}{l}\text { Idade } \\
\text { Milho } \\
\text { (dias) }\end{array}$} & \multirow{2}{*}{$\begin{array}{l}\mathrm{N}^{\circ} \text { Cigar. } \\
\text { por planta }\end{array}$} & \multicolumn{2}{|c|}{ Peso parte aérea ${ }^{1}(\mathrm{~g})$} & \multicolumn{2}{|c|}{ Peso raízes ${ }^{1}(\mathrm{~g})$} \\
\hline & & Verde & Seco & Verde & Seco \\
\hline \multirow[t]{4}{*}{10} & 0 & $79,9( \pm 19,37) \mathrm{a}$ & $8,6( \pm 2,51) \mathrm{a}$ & $71,9( \pm 9,03) \mathrm{a}$ & $7,1( \pm 1,40) \mathrm{a}$ \\
\hline & 1 & $62,9( \pm 19,31) b$ & $7,1( \pm 1,82) a b$ & $49,3( \pm 14,90) \mathrm{b}$ & $3,9( \pm 0,87) b$ \\
\hline & 5 & $52,9( \pm 6,64) b c$ & $5,8( \pm 0,99) b c$ & $47,6( \pm 10,28) b$ & $3,8( \pm 1,12) b$ \\
\hline & 10 & $47,7( \pm 3,81) \mathrm{c}$ & $5,1( \pm 0,38) \mathrm{c}$ & $33,6( \pm 2,36) \mathrm{c}$ & $2,7( \pm 0,14) \mathrm{c}$ \\
\hline \multirow[t]{4}{*}{15} & 0 & $79,4( \pm 11,14) \mathrm{a}$ & $8,4( \pm 2,28) \mathrm{a}$ & $47,0( \pm 7,83) a$ & $3,5( \pm 0,70) \mathrm{a}$ \\
\hline & 1 & $75,9( \pm 18,38)$ a & $8,0( \pm 1,24) a$ & $46,3( \pm 8,17) a$ & $3,2( \pm 0,58) \mathrm{a}$ \\
\hline & 5 & $70,3( \pm 12,11) \mathrm{a}$ & $7,5( \pm 1,81)$ a & $41,6( \pm 10,41) a b$ & $3,0( \pm 0,87) \mathrm{a}$ \\
\hline & 10 & $70,0( \pm 13,75) \mathrm{a}$ & $7,5( \pm 1,74)$ a & $37,6( \pm 9,80) b$ & $2,9( \pm 0,99) \mathrm{a}$ \\
\hline \multicolumn{2}{|c|}{ C. V. $(\%)$} & 13,5 & 15,1 & 11,6 & 16,5 \\
\hline
\end{tabular}

${ }^{1}$ Médias seguidas pela mesma letra, nas colunas e para cada idade de plântula, não diferem entre si pelo teste de Duncan $(\mathrm{P} \leq 0,05)$.

menor na cultura de sorgo que na de milho ou na área em pousio. A espécie de cigarrinha predominante nos ambientes estudados foi $D$. maidis e esta foi mais abundante nas áreas com milho do que nas demais. Plântulas de milho infestadas com $D$. maidis na densidade de 10 adultos/planta, sofreram redução, respectivamente, de 40 e $62 \%$ do peso seco da parte aérea e do sistema radicular.

\section{Agradecimentos}

O autor agradece ao Dr. Max de Menezes a identificação das cigarrinhas e ao técnico de laboratório Ronaldo G. Braga o apoio na condução dos ensaios.

\section{Literatura Citada}

Bushing, R. W. \& V. E. Burton. 1974. Leafhopper damage to silage corn in California. J. Econ. Entomol. 67:656-658.
Callahan, R. A., F. R. Holbook \& F. R. Shaw. 1966. A comparison of sweeping and vacuum collecting certain insects affecting forage crops. J. Econ. Entomol. 59: 478-479.

Campos, A. R., S. Gravena, R. Bertoso \& J. Barbieri. 1986. Artrópodes predadores na cultura algodoeira e comparação de métodos de amostragem. An. Soc. Entomol. Brasil 15:5-20.

Cherry, R. H., K.A. Wood \&W. G. Ruesink. 1977. Emergence trap and sweep net sampling for the potato leafhopper from alfalfa. J. Econ. Entomol. 70:279-282.

\section{Costa, A. S., E.W. Kitajima \& S.C.Arruda.} 1971. Moléstias de vírus e de micoplasma do milho em São Paulo. Rev. Soc. Bras. Fitopatologia 4:39-41. 
Douglas, W. A., W. A. Whitcomb, L. W. Hepner,V.W. M. Kirk \& R. Davis. 1966. Some Cicadellidae (Homoptera) collected from com in the southeastern United States. Ann. Entomol. Soc. Am. 59: 393-396.

Fazolin, M.. 1995. Levantamento de insetos e flutuação populacional de pragas que ocorrem na cultura do caupi (Vigna unguiculata (L.)Walp.) em Rio Branco (AC). Turrialba 45: 137-142.

Gallo, D., O. Nakano, S. Silveira Neto, R. P. L. Carvalho, G. C. Batista, E. Berti Filho, J. R. P. Parra, R.A. Zucchi \& S. B.Alves. 1978. Manual de entomologia agrícola.

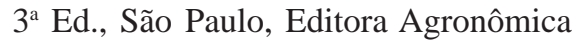
Ceres Ltda,531p.

Kitajima,E.W. 1979. Citopatologia e localização de vírus de milho e de leguminosas alimentícias nas plantas infectadas e nos vetores. Fitopat. Bras. 4: 241 - 254.

Kunkel, L. 0.1946. Leafhopper transmission of corn stunt. Proc. Nat. Acad. Sci. 32: 246-247.

Nault, L. R. \& E. D. Ammar. 1989. Leafhopper and planthopper transmission of plant viruses.Ann. Rev Entomol. 34: 503-529.

Raga, A., S. Gravena, S. A. de Bortoli, J. Arai, G. N. Wassano. 1990. Amostragem de insetos e atividade de artrópodes predadores na cultura do tomateiro de crescimento determinado. An. Soc. Entomol. Brasil 19:253-271.

Simonet, D. E., R. L. Pienkowski, D. G. Martinez \& R. D. Blakeslee. 1978. Laboratory and field evaluation of sampling techniques for the nynphal stage of the potato leafhopper on alfalfa. J. Econ. Entomol. 71: 840-842.

Stevens, L. M. \& A. L. Steinhauer. 1973. Evaluating the D-Vac> as a sampling tool for the alfalfa weevil adult. J. Econ. Entomol. 66:1328-1329.

Tsai, J. H., 1988. Bionomics of $D$. maidis (DeLong \& Wolcott), a vector of mollicutes and virus (Homoptera: Cicadellidae).p 209-221. In S. P. Maramorosch \& S. P. Raychaudhuri (ed.), Mycoplasma diseases of crops: basic and applied aspects, NewYork, Spring - Verlag, 456p.

Waquil, J. M., G. L. Teetes \& G. C. Peterson. 1986. Adult sorghum midge (Diptera: Cecidomyiidae) nonpreference for a resistant hybrid sorghum. J. Econ. Entomol. 79:455-458.

Recebido em 09/10/95. Aceito em 06/01/97. 